\title{
PARTON SPIN ASYMMETRIES IN POLARIZED HADRONS AT HIGH ENERGIES
}

\author{
Martin B. EINHORN \\ Randall Laboratory of Physics, University of Michigan, Ann Arbor, MI 48109-1120, USA \\ Jacques SOFFER ${ }^{1}$ \\ Department of Physics, Brookhaven National Laboratory, Upton, NY 11973, USA
}

Received 4 September 1985

\begin{abstract}
The predictions of perturbative QCD for the evolution of quark and gluon distributions for polarized hadrons, in general, and for polarized protons, in particular, are developed. Analytical and numerical studies suggest that quark and gluon polarizations increase with increasing energy at fixed Feynman $x$. Moreover, the average gluon asymmetry grows as $\ln Q^{2}$, receiving contributions from both quark and gluon asymmetries at lower energy. This has important implications for the growth of orbital angular momenta of partons and presents a challenge to model building. A new sum rule for a particular moment of the quark and gluon asymmetries is derived.

We conclude that more work is needed to determine how useful polarized proton beams might be in the search for and exploration of new physics in lepton-proton, $\mathrm{p} \overline{\mathrm{p}}$ and $\mathrm{pp}$ colliders.
\end{abstract}

\section{Introduction}

The predictions of QCD for the evolution of the unpolarized nucleon structure functions have been exhaustively studied [1]. Although a great deal of theoretical work has been devoted to spin physics at short distances [2], the polarized nucleon parton distributions are not well understood, owing in part to the scarcity of experimental data in a region of phase space where perturbative QCD can be confidently applied. In addition, there has not been overwhelming theoretical interest in the subject for a variety of reasons. At very high energy, the partons (quarks and gluons) are concentrated at low $x$ where the parton polarizations are expected to be small. Moreover, there is a widespread feeling that there is likely to be little correlation between the spin of the nucleon and the spin of the partons (i.e. parton asymmetries) at small $x$.

We have analyzed some of these issues in the context of perturbative QCD. In particular, we shall present analytical and computational arguments that certain parton polarizations grow with increasing energy (for fixed $x$ ).

1 Permanent address: Centre de Physique Théorique, CNRS-Luminy, Case 907, F-13288 Marseille Cedex 9, France. 
Our attitude is not that one needs polarized beams and/or targets to verify perturbative QCD. Rather, our concern is with the issue of whether polarized protons would provide a useful tool for the discovery and investigation of new physical phenomena, new particles or interactions, at very high energies. There is consensus that it is useful to have polarized lepton beams and, if quarks and gluons could be given large polarizations, undoubtedly it would be very desirable. However, for the reasons stated previously, there is considerable controversy about the utility of polarized hadrons. For an accelerator of a given energy, searches for new physical phenomena frequently focus not on the abundant events which dominate interactions, even at relatively large transverse momenta, but rather on the rare events of distinguishing signature, frequently associated with mass thresholds which would consume a substantial fraction of the available energy. For these high-mass events, the participating partons have relatively larger values of $x$ where the distributions are small but where polarization is apt to be relatively larger. Thus, the fact that certain parton polarizations at fixed $x$ increase with energy may be very useful, if the luminosity permits the exploration of the tails of the parton distributions. Polarization can provide information not obtainable in other ways and is a sensitive probe for new interactions which violate QCD symmetries such as parity.

Our new results, together with the historical importance of spin as a tool for unraveling the nature of new interactions, suggest that the question of the utility of polarization experiments at very high energy requires more careful analysis than have been performed so far. In this paper we will study the $Q^{2}$ evolution of the structure functions, reviewing first the unpolarized case in sect. 2 and then discussing the polarized case in sect. 3. Some of the experimental consequences of the highenergy behaviour of the parton spin asymmetries are examined in sect. 4 , and some concluding remarks are given in sect. 5 .

\section{Unpolarized distributions}

Let us consider the evolution equations for the parton distribution functions, for which an intuitive formulation has been provided by Lipatov [3] and by Altarelli and Parisi [4]. Let $q_{ \pm}^{i}(x, t)$ denote the quark distributions (of flavor $i$ ) in a polarized proton, either with helicity parallel $(+)$ or anti-parallel $(-)$ to the proton helicity. Similarly, $G_{ \pm}(x, t)$ denote the parallel $(+)$ and antiparallel $(-)$ gluon distributions. Here $t=\ln \left(Q^{2} / \Lambda^{2}\right)$, where $\Lambda$ is the QCD scale parameter and $Q$ is some momentum scale relevant to the particular process of interest. The usual distributions for unpolarized protons are the sums $q^{i}=q_{+}^{i}+q_{-}^{i}$ and $G=G_{+}+G_{-}$. In the following, we shall be particularly interested in the quark and gluon helicity asymmetries, $\Delta q=q_{+}-q_{-}$and $\Delta G=G_{+}-G_{-}$, but, to put this in context, let us first recall the general features of the $Q^{2}$ evolution of the unpolarized quark and gluon distributions.

It is well-known that, as $Q^{2}$ increases, the distributions grow for small $x$ and diminish for large $x$. However the $Q^{2}$ dependence is most easily expressed mathemati- 
cally in terms of the moments of the distributions, defined as

$$
f_{n}(t) \equiv \int_{0}^{1} \mathrm{~d} x x^{n-1} f(x, t),
$$

for any distribution $f(x, t)$. Given the moments, to obtain the distribution functions themselves, one must perform an inverse Mellin transform

$$
f(x, t)=\int_{-i \infty}^{i \infty} \frac{\mathrm{d} n}{2 \pi i} x^{-n} f_{n}(t),
$$

requiring analytic continuation to complex $n$. So it is useful to understand the behavior of the moments for noninteger $n$. The evolution equations are conveniently reviewed by Altarelli [5] whose notation we shall largely follow. (See, in particular, table 2, p. 40 of [5].) The various nonsinglet (e.g. valence) distributions obey

$$
\begin{aligned}
\frac{\mathrm{d}}{\mathrm{d} t} q^{\mathrm{NS}}(x, t) & =\frac{\alpha(t)}{2 \pi} \int_{x}^{1} \frac{\mathrm{d} y}{y} P_{\mathrm{qq}}\left(\frac{x}{y}\right) q^{\mathrm{NS}}(y, t) \\
& \equiv \frac{\alpha(t)}{2 \pi} P_{\mathrm{qq}} \otimes q^{\mathrm{NS}}
\end{aligned}
$$

with the strong coupling constant $\alpha(t)^{-1}=b t$, and $b \equiv(33-2 f) / 12 \pi$, where $f$ is the number of quark flavors (of mass less than $Q$ ). The solution for the moments is

$$
q_{n}^{\mathrm{NS}}\left(Q^{2}\right)=q_{n o}^{\mathrm{NS}} \mathrm{e}^{d_{n} S},
$$

where $d_{n} \equiv A_{n}^{\mathrm{qq}} / 2 \pi b$, and $A_{n}^{\mathrm{qq}}$ is the $n$th moment of $P_{\mathrm{qq} \cdot}$. We introduce the variable $S\left(Q^{2}\right)=\ln \left(t / t_{0}\right)$, where $t_{0}$ corresponds to some initial momentum value $Q_{0}$ where the distribution is presumed to be known. The exponent $d_{n}$ is negative (positive) for all real $n>1(n<1)$. The vanishing of $d_{1}$ implies that $q_{1}^{\mathrm{NS}}$ is energy independent, corresponding physically to the conservation of flavor quantum numbers. $A_{n}^{\text {qq }}$ has poles at nonpositive integers. The leading pole at $n=0$ can be shown to control the small $x$ behavior giving

$$
q^{\mathrm{NS}}(x, S)=q^{\mathrm{NS}}(x, 0)+2 R^{\mathrm{NS}} S \int_{x}^{1} \frac{\mathrm{d} y}{y} \frac{I_{1}\left(\zeta_{y}\right)}{\zeta_{y}} q^{\mathrm{NS}}\left(\frac{x}{y}, 0\right),
$$

where $I_{1}$ is the modified Bessel function of order one, $\zeta_{y} \equiv 2 \sqrt{R^{N S} S \ln (1 / y)}$, and $R^{\mathrm{NS}}$ is the residue of the pole of $d_{n}$ at $n=0 .\left(R^{\mathrm{NS}}=P_{\mathrm{qq}}(0) / 2 \pi b=2 / 3 \pi b\right)$. The small $x$ behavior of $q^{\mathrm{NS}}(x, 0)$ is believed to be related to Regge intercepts, with leading behavior proportional to $x^{-\alpha_{0}}$, corresponding to poles in $q_{n 0}$ at $n=\alpha_{0}$. For sufficiently small $x$, eq. (5) becomes

$$
q^{\mathrm{NS}}(x, S) \sim \sqrt{R^{\mathrm{NS}} S} \exp \left[2 \sqrt{R^{\mathrm{NS}} S \ln (1 / x)}\right] q^{\mathrm{NS}}(x, 0),
$$

The range of $x$ for which these approximations are valid is a complicated question. Corrections to eq. (5) are likely to be down by a power of $x$, whereas eq. (6) is 
good only for $\left[\ln (1 / x) / R^{N S} S\right] \gg 1$. The smallest value of $x$ permissible is more problematic, and we defer to extensive discussions of this subject [6]. The behavior of $q(x, S)$ at $x=1$ can also be deduced [7], but these predictions are not so relevant at very high energies and will not be reviewed here.

The singlet quark distribution $\Sigma(x, t)=\sum_{\text {flavors }}(q+\bar{q})$ and the gluon distribution $G(x, t)$ are coupled by the evolution equations, abbreviated as [5]

$$
\frac{\mathrm{d}}{\mathrm{d} t}\left(\frac{\Sigma}{G}\right)=\frac{\alpha(t)}{2 \pi}\left(\begin{array}{cc}
P_{\mathrm{qq}} & 2 f P_{\mathrm{qG}} \\
P_{\mathrm{Gq}} & P_{\mathrm{GG}}
\end{array}\right) \otimes\left(\begin{array}{c}
\Sigma \\
G
\end{array}\right),
$$

so their evolution is more complicated. The solution for the moments may be written as

$$
\begin{aligned}
\left(\begin{array}{c}
\Sigma_{n} \\
G_{n}
\end{array}\right)= & \frac{\Sigma_{n 0}+\left(a_{n}^{+}\right) G_{n 0}}{a_{n}^{+}-a_{n}^{-}}\left(\begin{array}{c}
-a_{n}^{-} \\
1
\end{array}\right) \exp \left[d_{n}^{+} S\right] \\
& +\frac{\Sigma_{n 0}+\left(a_{n}^{-}\right) G_{n 0}}{a_{n}^{+}-a_{n}^{-}}\left(\begin{array}{c}
a_{n}^{+} \\
-1
\end{array}\right) \exp \left[d_{n}^{-} S\right],
\end{aligned}
$$

where

$$
\begin{gathered}
a_{n}^{ \pm}=\frac{A_{n}^{\mathrm{GG}}-A_{n}^{\mathrm{qq}} \pm \Delta_{n}}{2 A_{n}^{\mathrm{Gq}}}, \quad \Delta_{n}=\sqrt{\left(A_{n}^{\mathrm{GG}}-A_{n}^{\mathrm{qq}}\right)^{2}+4 A_{n}^{\mathrm{qG}} A_{n}^{\mathrm{Gq}}} \\
d_{n}^{ \pm}=\frac{1}{4 \pi b}\left[A_{n}^{\mathrm{GG}}+A_{n}^{\mathrm{qq}} \pm \Delta_{n}\right] .
\end{gathered}
$$

We shall refer to $d_{n}^{+}\left(d_{n}^{-}\right)$as the dominant (subordinate) eigenvalue or exponent. (Unlike the original conventions of Altarelli and Parisi [4], a factor $2 f$ has been absorbed into $A_{n}^{q G}$.) These are complicated expressions, whose implications for the behaviors of the $x$-distributions are not so transparent. The dominant eigenvalue $d_{n}^{+}$is negative (positive) for $n>2(n<2)$. Its vanishing at $n=2$ corresponds to the conservation of momentum, $\Sigma_{2}+G_{2}=1$. To see this in the present formalism, one must note that conservation of momentum implies relations among the elements of the $A_{n}$ matrix [4], in particular $A_{2}^{q q}=-A_{2}^{G q}$. This implies $a_{2}^{+}=1$ so that, indeed, $\Sigma_{2}+G_{2}$ is conserved. (Below, we shall derive an analogous, hitherto unnoticed, sum rule for the singlet spin asymmetries.) Since $d_{2}^{-}<0$, one can predict the asymptotic momentum fractions $\Sigma_{2}$ and $G_{2}$ (as a function of the number of flavors).

These complicated expressions for the singlet moments do not yield obvious implications for the $x$-distributions. Roughly, $\Sigma(x, S)$ and $G(x, S)$ behave similarly with increasing $S$, falling at large $x$ but rising at small $x$. The precise value $x_{0}(t)$ at which the behavior crosses over is $Q^{2}$ dependent, with $x_{0}(t) \rightarrow 0$ as $t \rightarrow \infty$ so that, ultimately $x \Sigma$ and $x G$ become proportional to $\delta(x)$. This growth at small $x$ appears accentuated by the fact that $\Sigma$ and $G$ are proportional to $x^{-1}$ as $x \rightarrow 0$. Equations similar to eq. (5) for the nonsinglet distribution can be derived, with approximate 
behavior as $x \rightarrow 0$, given by

$$
x G(x, S) \propto \exp \left[2 \sqrt{R_{+} S \ln (1 / x)}\right],
$$

where $R_{+}$is the residue of the pole of $d_{n}^{+}$at $n=1$. This is in fact proportional to the pole in $A_{n}^{\mathrm{GG}}$, and it turns out that $R_{+}=C_{2}(G) / \pi b=3 / \pi b$, a number of order 1. The coefficient of the exponential is a power of $\ln (1 / x)$ which depends on the precise behavior of $x G(x, O)$ as $x \rightarrow 0 . x \Sigma$ is slightly less singular as $x \rightarrow 0$, with $x \Sigma \propto\left[S / R_{+} \ln (1 / x)\right]^{1 / 2} x G$. Since the range of validity of this approximation requires $\left[\ln (1 / x) / R_{+} S\right] \gg 1, x \Sigma \ll x G$. Thus gluons ultimately dominate quarks in those processes in which both participate, such as in hadron scattering.

This completes our review of the properties of the unpolarized distributions.

\section{Polarized distributions}

We shall now carry through an analogous discussion for the helicity asymmetries $\Delta q$ and $\Delta G$. The nonsinglet (valence) quark distributions, $\Delta q^{\mathrm{NS}}(x, t)$, obey exactly the same equation, eq. (3), as $q^{\mathrm{NS}}$, because of helicity conservation of the coupling of quarks to vector gluons. Note in particular that $\Delta q_{1}^{\mathrm{NS}}$ is a constant, corresponding to a conserved average spin asymmetry for each flavor of quark. The behavior of $\Delta q^{\mathrm{NS}}(x, 0)$ as $x \rightarrow 0$ is believed to be similar also, so the valence quark asymmetry evolves like $q^{\mathrm{NS}}$.

Can anything more precise be said about the evolution of the valence quark polarization $\Delta q^{\mathrm{NS}}(x, S) / q^{\mathrm{NS}}(x, S)$ ? This polarization is a rough measure of the contribution of valence quarks to the asymmetry of certain physical processes, such as parity violation in jet production or the polarization of $W$ and $Z$ bosons if produced in polarized $\mathrm{p} \overline{\mathrm{p}}$ or $\mathrm{pp}$ collisions. Intuitively and experimentally [8], the quark polarization at low energy is large at large $x$ and small at small $x$. Since the valence quark evolution with energy is due to helicity conserving gluon emission, one would expect to find the valence quark asymmetry conserved ( $\Delta q_{1}$ is constant) but evolving to lower values of $x$. Thus, we expect the valence quark polarization at fixed $x$ to increase with energy. We now provide a formal proof of this: From the evolution equations, one can show that

$$
\frac{\mathrm{d}}{\mathrm{d} S} \frac{\Delta q(x, S)}{q(x, S)}=\frac{1}{2 \pi b} \int_{x}^{1} \frac{\mathrm{d} y}{y} P_{\mathrm{qq}}\left(\frac{x}{y}\right) \frac{q(y, S)}{q(x, S)}\left[\frac{\Delta q(y, S)}{q(y, S)}-\frac{\Delta q(x, S)}{q(x, S)}\right] .
$$

One need not regulate the singularity in $P_{\mathrm{qq}}(z)$ at $x=1$, because the quantity in brackets vanishes for $x=y$. Thus the kernel

$$
P_{\mathrm{qq}}(z)=\frac{4}{3} \frac{1+z^{2}}{1-z}
$$

is strictly positive. So long as the polarization of valence quarks is monotonically increasing with $x$, i.e. 


$$
\frac{\Delta q(y, S)}{q(y, S)}>\frac{\Delta q(x, S)}{q(x, S)} \quad \text { for } y>x,
$$

the integrand is manifestly positive. Thus, the derivative $(\mathrm{d} / \mathrm{d} S) \Delta q / q>0$, so the valence quark polarization increases with increasing energy! Certainly all models compatible with the SLAC-Yale data [8] must have the valence quark polarization strongly correlated with the nucleon spin, increasing with increasing $x$. Although we have not proved this monotonicity persists at all energies, it is certainly plausible and is supported by numerical integration of this equation. In fig. 1, we show the results on $\Delta V / V$ for the valence quark distributions considered in a recent study [9]. It is interesting to understand better the origin of this increase, so we show in fig. 2 the separate evolution of $\Delta V$ and $V$. Note that over this range in $Q^{2}$, for the interval $0.08<x<0.18, \Delta V$ actually increases while $V$ decreases. Although it becomes increasingly difficult to probe high $x$ for large $Q^{2}$, we note that the new ep collider HERA promises to determine the unpolarized structure functions with reasonable accuracy at $Q^{2}=10^{4}(\mathrm{GeV} / \mathrm{c})^{2}$ out to about $x=0.5$. Should a polarized proton beam be developed at some future date, experimenters would be able to verify this increase in quark polarization at fixed $x$, an unambiguous prediction of QCD.

Since $\Delta q^{\mathrm{NS}} / q^{\mathrm{NS}}$ increases with energy if the initial polarization increases with $x$, it is theoretically amusing to consider its ultimate fate since $\Delta q^{\mathrm{NS}} / q^{\mathrm{NS}} \leqslant 1$. Noting

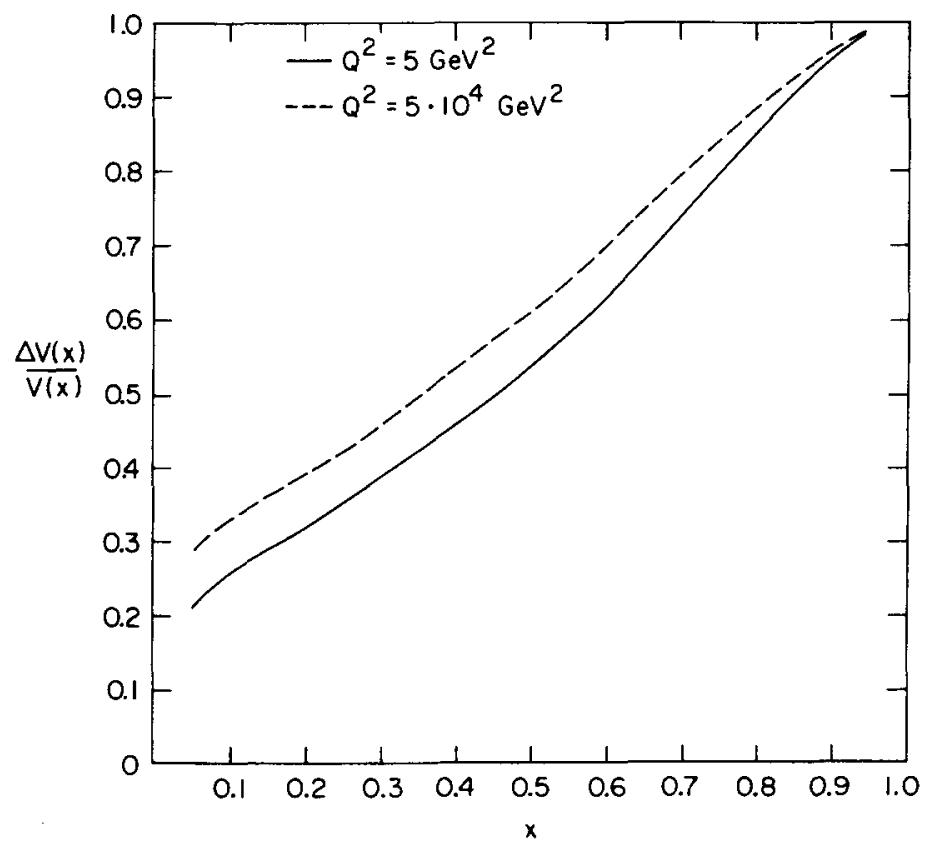

Fig. 1. Valence quark polarization $\Delta V(x) / V(x)$ as a function of $x$ for $Q^{2}=5 \mathrm{GeV}^{2}$ (solid curve) and $Q^{2}=5 \times 10^{4} \mathrm{GeV}^{2}$ (dashed curve) from ref. [9]. 


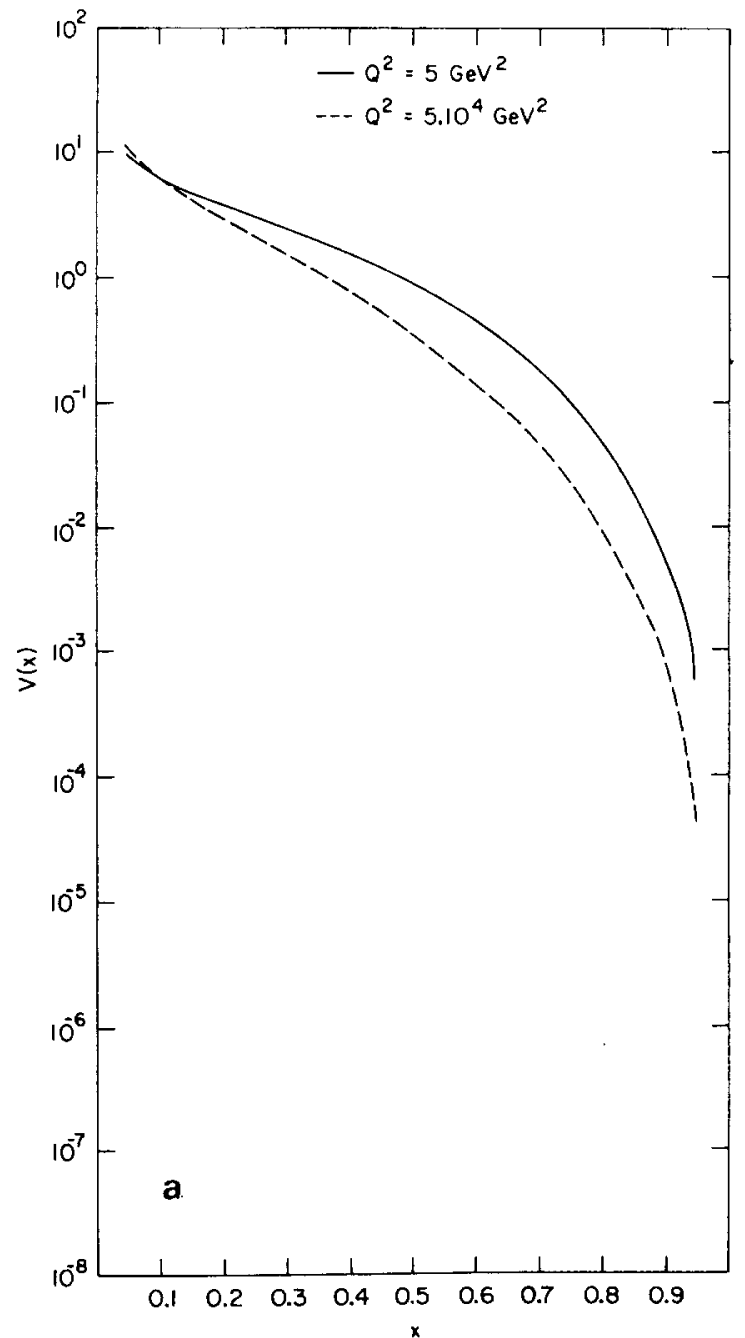

Fig. 2a. Valence quark distribution $V(x)$ as a function of $x$ for $Q^{2}=5 \mathrm{GeV}^{2}$ (solid curve) and $Q^{2}=$ $5 \times 10^{4} \mathrm{GeV}^{2}$ (dashed curve) from ref. [9].

that a polarization which is a constant function of $x$ is a stable solution, we conjecture that

$$
\lim _{Q^{2} \rightarrow \infty} \frac{\Delta q^{\mathrm{NS}}\left(x, Q^{2}\right)}{q^{\mathrm{NS}}\left(x, Q^{2}\right)}=\lim _{x \rightarrow 1} \frac{\Delta q^{\mathrm{NS}}\left(x, Q_{0}^{2}\right)}{q^{\mathrm{NS}}\left(x, Q_{0}^{2}\right)} .
$$

Even though both $\Delta q^{\mathrm{NS}}\left(x, Q^{2}\right)$ and $q^{\mathrm{NS}}\left(x, Q^{2}\right)$ tend to zero at fixed $x>0$, this conjecture, if proved, would certainly contradict the prevalent notion that quark polarization is degraded at high energy.

Turning to the more complicated singlet helicity asymmetries, one finds equations of exactly the same form as eq. (7) with solutions of the same form as eq. (8). (One 


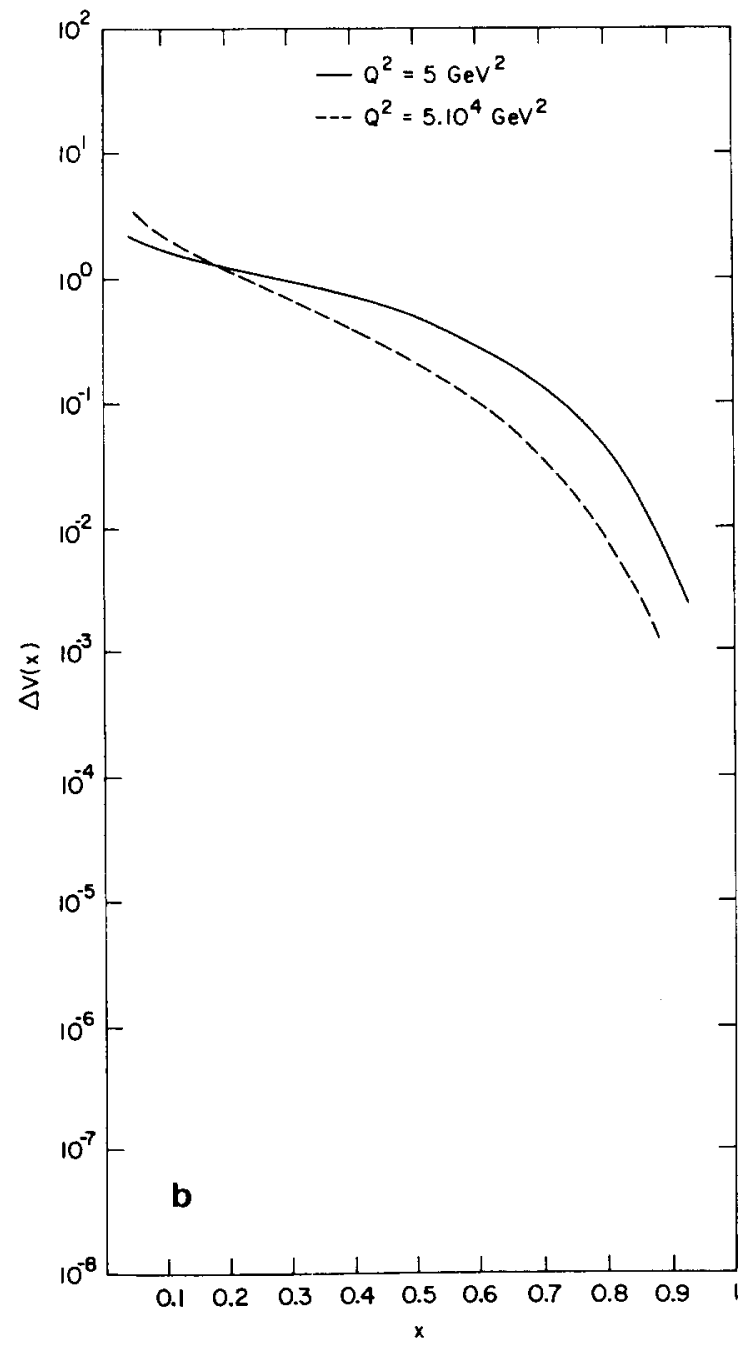

Fig. 2b. Same as Fig. 2a for the valence quark asymmetry $\Delta V(x)$.

TABLE 1

Numerical values of $\nu$ corresponding to $\Delta d_{\nu}^{+}=0$ for different number of flavors and coefficients of the general solution for the moments of polarized structure functions.

\begin{tabular}{lllll}
\hline$f$ & $\nu$ & $\Delta a_{\nu}^{+}$ & $\Delta a_{\nu}^{-}$ & $\Delta d_{\nu}^{-}$ \\
\hline 3 & 1.57 & 1 & -0.36 & -0.36 \\
4 & 1.54 & 0.94 & -0.49 & -0.41 \\
5 & 1.52 & 0.88 & -0.62 & -0.48 \\
6 & 1.49 & 0.84 & -0.76 & -0.57 \\
\hline
\end{tabular}


simply replaces the distributions $\Sigma, G$, and the kernels of $P$ by the asymmetries $\Delta \Sigma$, $\Delta G$, and $\Delta P$, respectively.) The dominant exponent $\Delta d_{n}^{+}$is negative (positive) for $n>\nu,(n<\nu)$, where the value of $\nu$ lies in the interval, $1<\nu<2$, the precise value depending weakly on the number of flavors. (See table 1.)The vanishing of the dominant exponent $\Delta d_{\nu}^{+}=0$ leads to a conserved quantity similar to momentum conservation for the unpolarized distribution. Thus

$$
\Delta \Sigma_{\nu}+\left(\Delta a_{\nu}^{+}\right) \Delta G_{\nu}
$$

is independent of $Q^{2}$, a $\nu$ sum rule for spin asymmetries. As can be seen in table $1, \Delta a_{\nu}^{+}=1$ for $f=3$, and in this case we have exactly

$$
\Delta A_{\nu}^{\mathrm{qq}}=-\Delta A_{\nu}^{\mathrm{Gq}}, \quad \Delta A_{\nu}^{\mathrm{qG}}=-\Delta A_{\nu}^{\mathrm{GG}} .
$$

$\Delta a_{\nu}^{+}$decreases from this value for increasing number of flavors. Because the subordinate eigenvalue.is negative, one can predict the àsymptotic values of the singlet quark and gluon moments

$$
\left(\begin{array}{c}
\Delta \Sigma_{\nu} \\
\Delta G_{\nu}
\end{array}\right) \rightarrow \frac{\Delta \Sigma_{\nu 0}+\left(\Delta a_{n}^{+}\right) \Delta G_{\nu 0}}{\Delta a_{\nu}^{+}-\Delta a_{\nu}^{-}}\left(\frac{-\Delta a_{\nu}^{-}}{1}\right) .
$$

The asymptotic ratio of the quark to gluon moments can be read from table 1 . However, the small value of the subordinate exponent $\Delta d_{v}^{-}$implies very slow approach to the asymptotic value so one should not be surprised if deviations are large in practice. Regardless, the sum rule should be valid and should provide a useful check on numerical computations at the very least. Because the singlet quark and gluon asymmetries are believed to be nearly constant as $x \rightarrow 0$, this sum rule should not be sensitive to the small $x$ behavior. The existence of this sum rule is a detailed dynamical prediction of QCD.

The subordinate exponent $\Delta d_{n}^{-}$vanishes at $n=1$, as is well known. A priori this would not be so useful, but, since the quark asymmetry $\Delta \Sigma_{1}$ decouples from the dominant eigenvalue $\left(\Delta a_{1}^{-}=0\right)$, this has some utility. The constancy of $\Delta \Sigma_{1}$ is well-known [10] and corresponds to helicity conservation owing to the vector nature of the gluon coupling. The behavior of $\Delta G_{1}$ has received less attention, even though the positivity of the dominant eigenvalue implies the average gluon asymmetry increases with energy. In fact, $\Delta d_{1}^{+}=1$ so that

$$
\Delta G_{1} \rightarrow\left(\Delta G_{10}+\frac{1}{\Delta a_{1}^{+}} \Delta \Sigma_{10}\right) \frac{t}{t_{0}}
$$

as $t=\ln \left(Q^{2} / \Lambda^{2}\right) \rightarrow \infty$. Note that the growth is driven in part by the initial quark asymmetry, so that this conclusion follows regardless of how small $G_{10}$ might be. (The coefficient of $\Delta \Sigma_{10},\left(\Delta a_{1}^{+}\right)^{-1}=\pi b$, is not particularly small.) However interesting this result may be, we wish to emphasize that the quark asymmetry $\Delta \Sigma_{n}$ couples to the dominant eigenvalue for $n \neq 1$. Since $\Delta d_{n}^{+}>0$ for $n<\nu$, both the quark and the gluon singlet moments are increasing with energy. 
Conservation of angular momentum along the direction of motion of the nucleon takes the form

$$
\frac{1}{2}=\frac{1}{2} \Delta \Sigma_{1}+\Delta G_{1}+\left\langle L_{z}\right\rangle,
$$

where $\left\langle L_{z}\right\rangle$ represents the contribution of the orbital angular momentum of the partons. It has been noted previously that the neglect of the orbital term is inconsistent with the evolution equations [10], but our observation that $\Delta G_{1}$ increases proportional to $\ln Q^{2}$ underscores the importance of $\left\langle L_{z}\right\rangle$, which necessarily must compensate this growth. Since

$$
L_{z}=\left(\boldsymbol{r}_{\mathrm{T}} \times \boldsymbol{p}_{\mathrm{T}}\right)_{z},
$$

unlike in the parton model, transverse momenta cannot be ignored at high energies. This is indeed the case in perturbative QCD where the cut off in $p_{\mathrm{T}}$ is known to be of the order of $Q[3,4]$. Models of nucleon asymmetries universally neglect orbital excitations, so they can at best be approximately valid below a certain energy scale. More work is needed to determine this range of validity and to understand better the interdependence of quark asymmetries and gluon asymmetries. The small $x$ behavior can also be analyzed in a manner similar to the unpolarized case. One finds that

$$
\Delta G(x, S) \sim \exp \left[2 \sqrt{\Delta R_{+} S \ln (1 / x)}\right]
$$

up to powers of $\ln (1 / x)$. Here $\Delta R_{+}$is the residue of the pole of $\Delta d_{n}^{+}$at $n=0$, given by

$$
\Delta R_{+}=\frac{1}{3 \pi b}[10+\sqrt{64-6 f}] .
$$

Although $\Delta G$ does not have the power of $x^{-1}$ present in $G$, it is amusing that the exponential factor has a larger coefficient than in the unpolarized case, i.e., $\Delta R_{+}>R_{+}$. As an example, for $f=6$ flavors, $\Delta R_{+}=2.91$ whereas $R_{+}=1.71$. Thus

$$
\frac{\Delta G}{G} \sim x \exp [0.80 \sqrt{S \ln (1 / x)}] .
$$

One sees that the gluon polarization $(\Delta G / G)$ remains small at small $x$, although the effective power of $x$ may be reduced from 1 . It is interesting that once again, we have found a polarization which increases with energy (at fixed $x$ ) within this limited range of validity. Since $\Delta \Sigma$ behaves like $\Delta G$ as $x \rightarrow 0$, the preceding remarks apply all the more to the singlet quark distribution.

Can one establish results similar to the non-singlet result concerning the correlation of the $x$-dependence of the quark and gluon polarizations at low energy with their energy evolution? Unfortunately, this seems to be a complicated question involving not only the behavior of these distributions but also the nature of the probability functions $P$ and $\Delta P$. The point is that, for gluons, there are nonzero amplitudes for both helicity states regardless of whether the gluon arises from a quark or gluon 
branching. One sees in the explicit solutions for each moment, of the form of eq. (8), a mixing of quark and gluon moments in the eigenvectors. As an indication of the complexity, consider the purely gluonic case, neglecting fermions altogether. The gluon polarization can be easily shown to obey

$$
\begin{aligned}
\frac{\mathrm{d}}{\mathrm{d} S} \frac{\Delta G(x, S)}{G(x, S)}= & \frac{1}{2 \pi b} \int_{x}^{1} \frac{\mathrm{d} y}{y} \frac{G(y)}{G(x)}\left\{P_{++}^{\mathrm{GG}}\left(\frac{x}{y}\right)\left[\frac{\Delta G(y)}{G(y)}-\frac{\Delta G(x)}{G(x)}\right]\right. \\
& \left.-P_{-+}^{\mathrm{GG}}\left(\frac{x}{y}\right)\left[\frac{\Delta G(y)}{G(y)}+\frac{\Delta G(x)}{G(x)}\right]\right\} .
\end{aligned}
$$

The first term in the integrand, the helicity conserving term, is of the same form as in the nonsinglet quark case, but the second term, due to helicity flip, is manifestly negative. As a result, it is difficult to draw model independent conclusions. Perhaps it is useful to note that $P_{++}^{G G}(z)>P_{-+}^{G G}(z)>0$ for $0.4 \leqslant z<1$. So for large $x$, we can perhaps neglect the second term and the form reduces to one similar to the nonsinglet case. Thus our conclusion is the same: If the gluon polarization is monotonically increasing with $x$, it will increase with energy, for large $x$ (say $x>0.5$ ). This conclusion is unlikely to be altered by the addition of the singlet quark distributions, $\Sigma$ and $\Delta \Sigma$, provided the quark polarization is also monotonically increasing and the quark polarization has the same sign as the gluon polarization.

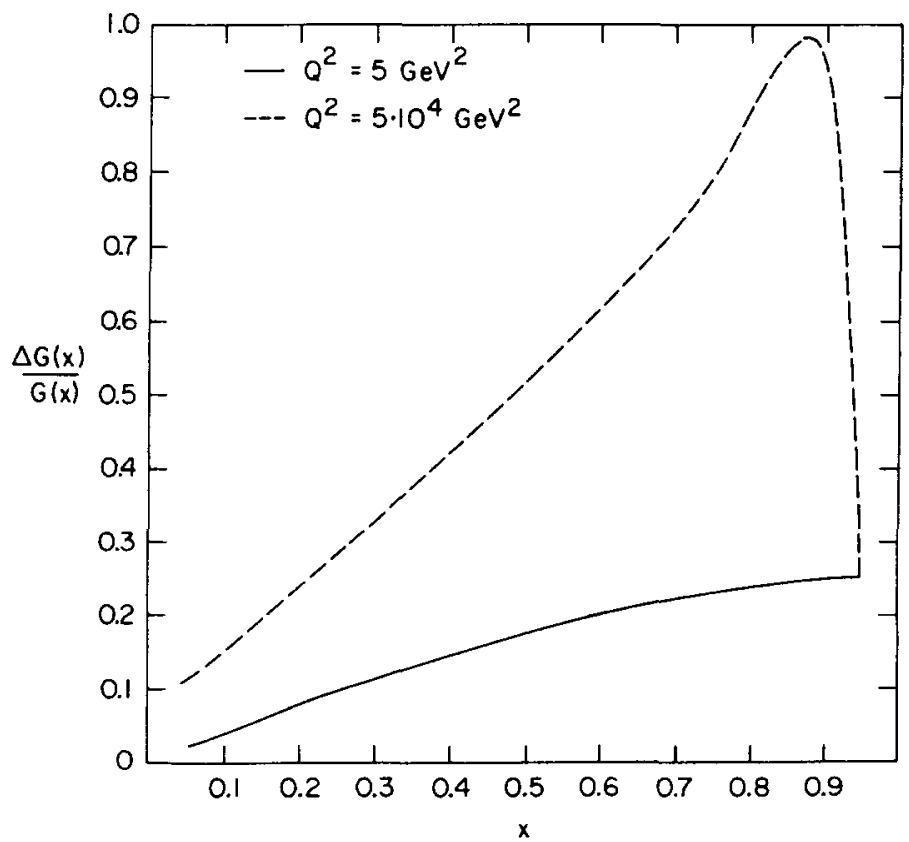

Fig. 3. Gluon asymmetry $\Delta G(x) / G(x)$ as a function of $x$ for $Q^{2}=5 \mathrm{GeV}^{2}$ (solid curve) and $Q^{2}=5 \times 10^{4}$ $\mathrm{GeV}^{2}$ (dashed curve) from ref. [9]. 
Explicit numerical solutions to the coupled equations in a recent model [9] lend quantitative support to the notion that the gluon polarization at fixed $x$ may rise dramatically with energy. In fig. 3, we reproduce a figure from this work demonstrating a very substantial growth in $(\Delta G / G)$ at all $x$. In our view, there is considerable uncertainty about the magnitude of the intial gluon asymmetry, for which there are no direct experimental or theoretical constraints except that $|\Delta G| \leqslant G$. The important conclusion is the rise of gluon polarization with energy ${ }^{\star}$. Further insight into the origin of this effect is provided by fig. 4 , where the behavior of $G$ and $\Delta G$ separately

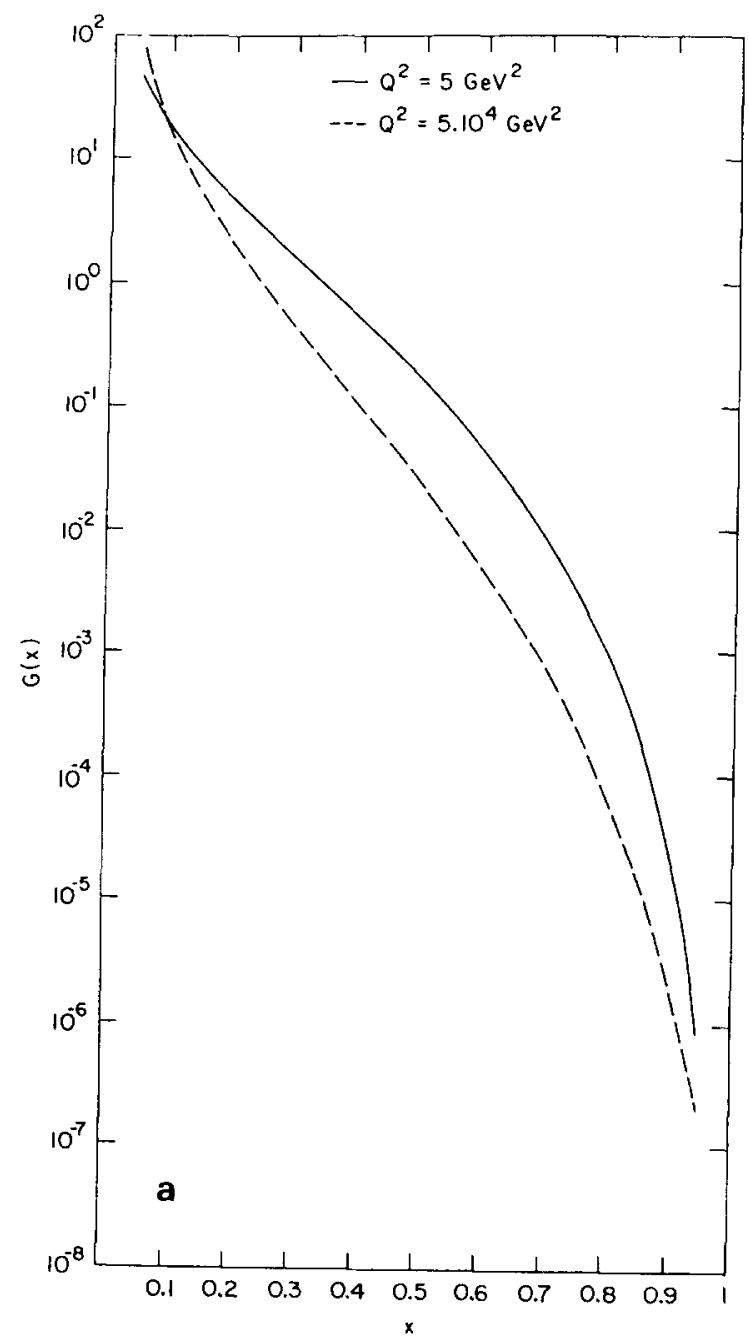

Fig. 4a. Gluon distribution $G(x)$ as a function of $x$ for $Q^{2}=5 \mathrm{GeV}^{2}$ (solid curve) and $Q^{2}=5 \times 10^{4} \mathrm{GeV}^{2}$ (dashed curve) from ref. [9].

* In this work, the number of effective flavors was taken to be $f=3$ for all $Q^{2}$. The effects of heavier quarks on these results will be investigated in future work. 


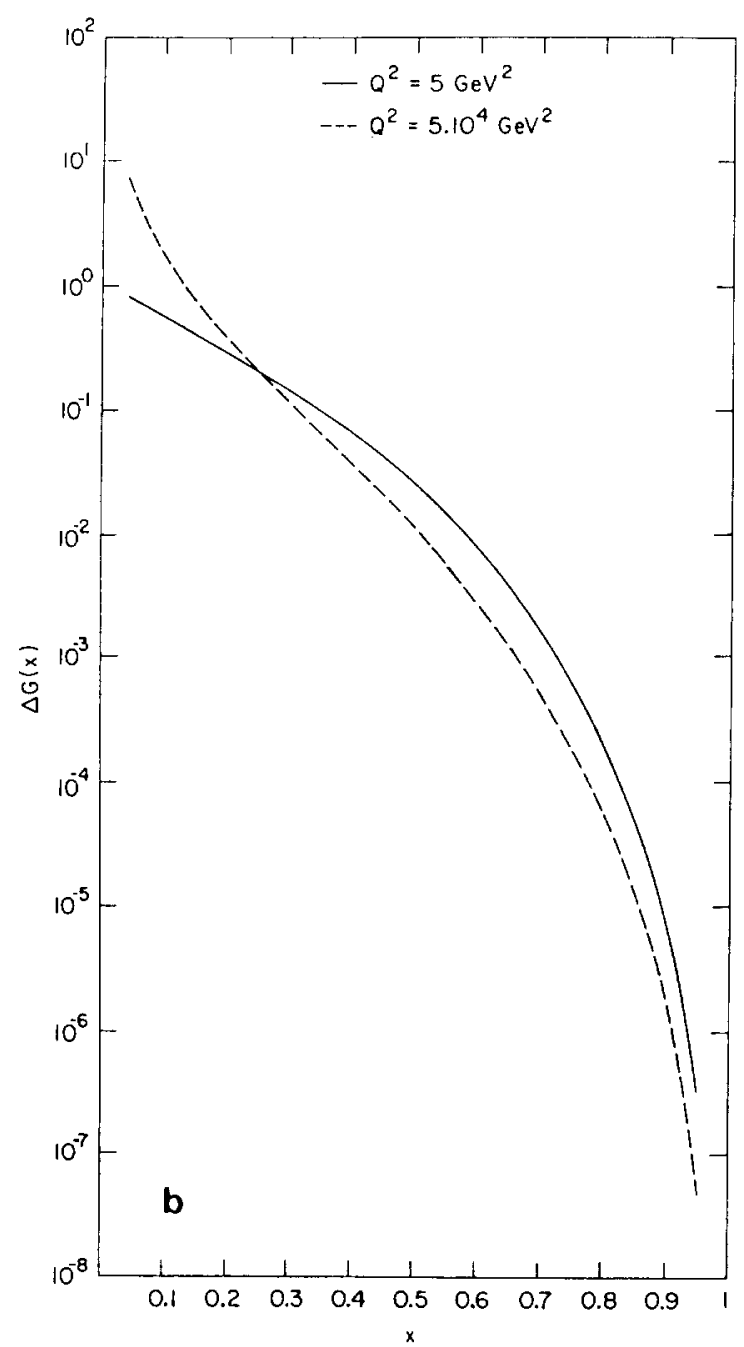

Fig. 4 b. Same as fig. $4 \mathrm{a}$ for the gluon asymmetry $\Delta G(x)$.

are shown. Note that over this energy range, in the interval $0.10<x \leqslant 0.25, \Delta G$ increases while $G$ decreases. While it is not an easy matter to ascertain the dominant origin of these effects, we believe it is largely due to the transfer of the substantial low-energy quark asymmetry into the high-energy gluon asymmetry.

\section{Experimental prospects and implications}

We repeat that models tend to minimize possible gluon polarization and that there is no direct experimental information on this quantity. As a result, it is worthwhile to ask which experiments would be sensitive to such polarization. Noting that wide-angle jet production is dominated by gluon-gluon or quark-gluon scattering out to be very large $p_{\mathrm{T}}$, measurements of angular asymmetries with polarized 
beams and targets may offer one source of information. We recall that wide angle gluon-gluon scattering is largely helicity conserving, which enhances the prospects. (See fig. 5.10 of [2].) Heavy quark production (at least for bottom and top) may be dominated by gluon-gluon interactions in hadron-hadron collisions and by photongluon fusion in lepton-hadron collisions. The sensitivities of these reactions to polarization have yet to be worked out. Prompt photon production at large $p_{\mathrm{T}}$ appears to be one of the cleanest sources of information on gluon distribution functions. It is also an efficient transmitter of the gluon polarization. In fig. 5 , we show the double helicity asymmetry in prompt photon production at fixed longitudinal momentum fraction $x_{F}$ as a function of the transverse momentum fraction $x_{\mathrm{T}}$ in the model of ref. [9]. We regard the absolute value of this asymmetry as quite uncertain but the increase as a function of $Q^{2}$ exemplifies the effect of the evolution equations and is quite encouraging. Notice that the asymmetry is far greater at large $x_{\mathrm{F}}$ than at $x_{\mathrm{F}}=0$. Unfortunately at SSC energy the cross section at $x_{\mathrm{T}}=0.1$ for $x_{\mathrm{F}}=0$ is expected to be of the order of $10^{-40} \mathrm{~cm}^{2} / \mathrm{GeV}$ and to decrease for larger $x_{\mathrm{F}}$ values. This makes the test very hard so perhaps one should also considered single jet production at high $p_{\mathrm{T}}$ which has a similar asymmetry with a much larger cross section. Anyway it would be very helpful to have a good measurement of prompt photon production with polarized proton beams on a polarized target in a range of $Q^{2}$ where perturbative QCD is trustworthy. Obviously, this places great demands on the initial state polarizations and luminosity. Although more theoretical work needs to be done, in the absence of reliable methods for predicting structure functions from nonperturbative QCD, there is no alternative to obtaining direct experimental information on the gluon polarization in a proton.

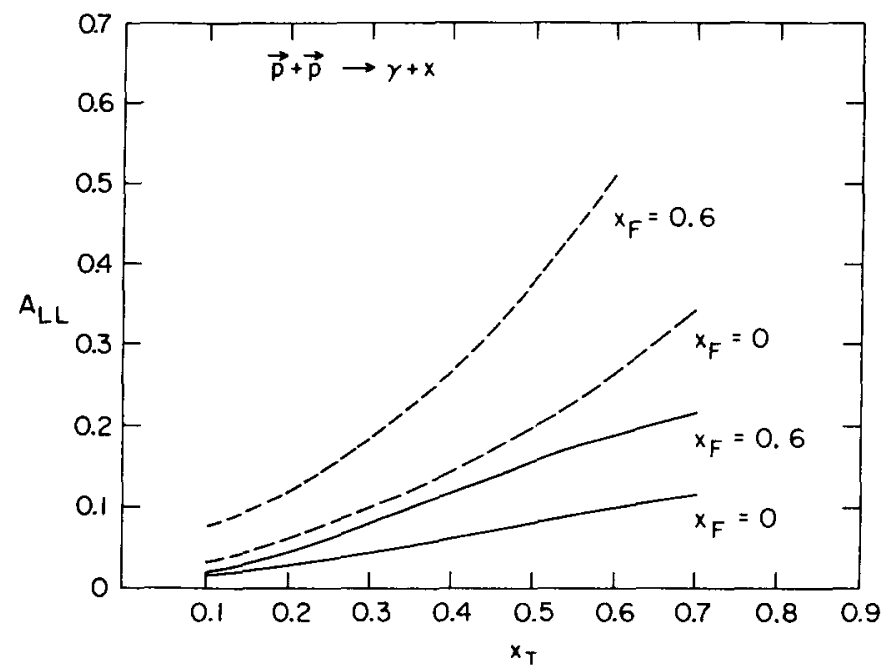

Fig. 5. Predictions based on ref. [9] for the double helicity asymmetry $A_{\mathrm{LL}}$ in $\overrightarrow{\mathrm{p}} \overrightarrow{\mathrm{p}} \rightarrow \gamma \mathrm{X}$ at $\sqrt{s}=40 \mathrm{TeV}$ for fixed $x_{\mathrm{F}}$ as a function of $x_{\mathrm{T}}$ with $Q^{2}=5 \mathrm{GeV}^{2}$ (solid curves) and with the evolution scale $Q^{2}=\frac{1}{4} p_{\mathrm{T}}^{2}$ (dashed curves). 
For quark asymmetries, the outlook is somewhat more hopeful, inasmuch as the European Muon Collaboration will soon report results of muon scattering from a polarized target at the SPS [11].

There are other QCD predictions of significance for polarization experiments, because the fragmentation functions characterizing the probability that a quark or gluon yields a hadron also obey these evolution equations [12]. This is currently under investigation and will be reported elsewhere [13]. Effects occur similar to those found for the structure functions, viz., with increasing $Q^{2}$, the fragmentation of a quark or a gluon into a spinning hadron shows the hadron's helicity strongly correlated, with the average asymmetry growing as $\ln Q^{2}$. This could be of great significance in the production of "self-analyzing" particles such as the hyperons or for charm, bottom, or top jets or particles.

\section{Conclusions}

We have demonstrated that, in a number of cases, polarization effects may increase with energy. We wish to emphasize that the general conclusions reached concerning the polarized proton structure functions would be true for any polarized hadron. One can imagine many related questions, for example, what could be learned from studies on quarkonium states of definite helicity? Would polarization be an aid in the determination of the glueball spectrum? Should hints of supersymmetry be revealed in accelerator experiments, how would polarization help resolve the properties of the new particles? It has been pointed out that the use of polarized beams in $\mathrm{e}^{+} \mathrm{e}^{-}$collisions at high energies must lead to spectacular effects for the production of SUSY particles [14]. There are also interesting predictions for jets and for gauge boson production in hadronic collisions [15]. On the other hand, if technicolor ideas are correct, there are also particles such as leptoquarks and color octet scalars $\left(P_{8}\right.$ or $\left.\eta_{\mathrm{T}}\right)$ for which polarization studies may be useful.

The notion that parton asymmetries are diluted by the rapid growth in parton multiplicities is possibly a misleading oversimplification of the prospects for polarization experiments at high energy. The multiplicity growth is driven by the extremely small $x$ behavior whereas our primary physics interests, especially in searching for and exploring new phenomena, may involve structure functions at relatively larger values of $x$. Thus, for a multi-TeV collider, while polarization may be insignificant at small $x$ (say $x \leqslant 0.01$, which region is responsible for the bulk of the events, our interest may be on the relatively rarer phenomena involving partons at larger values of $x$ (say, $x \geqslant 0.05$ ) where polarization may be more substantial. This is all the more likely if the new phenomena violate one of the natural symmetries of QCD, such as parity. We have offered no firm conclusion at this time, since much more work is needed if the issue is to be definitively resolved.

As plans proceed for the next generation of hadron colliders, these questions become all the more urgent. Given our present ignorance of the breakdown to be 
expected of the standard model, would it not be prudent to design future accelerators keeping in mind that, at some time in the future, the lattice may have to be modified to provide for the acceleration of polarized protons? We understand that this may require more and longer straight sections, planar rather than undulating rings, and perhaps larger tunnels. Might these requirements not be much more inexpensively accommodated if provided for in the initial design of the unpolarized beams?

A fundamental step in obtaining a better understanding of the structure of spinning protons is a determination of the quark structure functions for polarized protons. To this end, a polarized proton beam at HERA would provide an incomparable facility, perhaps never to be duplicated.

This work has been partly supported by the US Department of Energy under contract nos. DE-AC02-76CH00016 and DE-AC02-76ER01112.

We wish to thank participants of the Workshop on Polarized Beams for the SSC for stimulating our interest in these questions. One of us (M.B.E.) wishes to thank members of the Oregon Workshop on Super High Energy Physics for discussions, in particular, J.C. Collins, G. Martinelli, F. Paige, E. Reya, and Wu-Ki Tung. He would also like to thank its Director, D. Soper, for his interest and hospitality during the completion of this work. J.S. wishes to acknowledge the hospitality of the theoretical groups at Argonne and Brookhaven National Laboratories, where portions of this work were performed. Finally, we are indebted to P. Chiappetta and $\mathrm{J}$. $\mathrm{Ph}$. Guillet in Marseille, for providing us with additional computational results of the model of ref. [9].

\section{Note added}

While completing this paper, we received two related preprints [16]. In the first, the authors extend the Altarelli-Parisi equations to the supersymmetric extension of the standard model. They have also noted the growth of $\Delta G_{1}$, which is determined by the initial values of the gluon and singlet quark moment, as in the nonsupersymmetric case. (Just as in the standard model, their results imply $\Delta G_{1} \propto \ln Q^{2}$, again suggesting a simple physical origin for this common result.)

In the second paper, the authors express concern about this growth of $\Delta G_{1}$. They propose an adhoc modification of $\Delta P_{\mathrm{Ga}}$ for $a=$ quark, gluon (or gluino in the supersymmetric extension) by adding a term $\beta_{a} \delta(z)$, whose coefficient is determined by the requirement that the spin sum rule eq. (17) be satisfied without the addition of orbital angular momentum. They suggest that the growth of orbital angular momentum is somehow inconsistent with the small $p_{\mathrm{T}}$ assumed by the parton model. On the contrary, one should recall that, in the Lipatov-Altarelli-Parisi derivation $[3,4]$, the evolution with $Q^{2}$ stems from the $\log p_{\mathrm{T}}$ growth (with $p_{\mathrm{T}} \sim Q$ ) of the branching probabilities. Here is precisely where QCD differs from the naive parton model and exact scaling. The behavior of the distribution functions for very small 
$x$ requires the inclusion of higher order corrections and remains an active subject for research [17]. The behavior as $x \rightarrow 0$ is quite unlike the singularities of the Altarelli-Parisi kernels as $x \rightarrow 1$, where the addition of a $\delta(1-x)$ term is required by wave function renormalization. Finally, the authors addition of a $\delta(z)$ term introduces a peculiar non-analyticity into the $\Delta A_{n}$ moments. As noted in our text, the gluon moment $\Delta G_{n}$ is growing for all $n<\nu$. Its cancellation precisely at $n=1$ does not change this fact. In short, we believe the proposed modification is neither motivated by nor consistent with what we know of QCD.

\section{References}

[1] E. Eichten, I. Hinchliffe, K. Lane and C. Quigg, Rev. Mod. Phys. 56 (1984) 579

[2] N.S. Craigie, K. Hidaka, M. Jacob and F.M. Renard, Phys. Reports 99 (1983) 69, and references therein

[3] L.N. Lipatov, Sov. J. Nucl. Phys. 20 (1975) 94

[4] G. Altarelli and G. Parisi, Nucl. Phys. B126 (1977) 298

[5] G. Altarelli, Phys. Reports 81 (1982) 1

[6] L.V. Gribov, E.M. Levin and M.G. Ryskin, Nucl. Phys. B188 (1981) 555; Phys. Reports 100 (1983) 1 ;

A. Bassetto, M. Ciafaloni and G. Marchesini, Phys. Reports 100 (1983) 201;

A. Bassetto, M. Ciafaloni, G. Marchesini and A.H. Mueller, Nucl. Phys. B207 (1982) 189

[7] D.J. Gross, Phys. Rev. Lett. 32 (1974) 1071; in Methods in field theory, ed. R. Balian and J. Zinn-Justin (North-Holland, Amsterdam, 1976)

[8] M.J. Alguard et al., Phys. Rev. Lett. 37 (1976) 1258, 1261; 41 (1978) 70; G. Baum et al., Phys. Rev. Lett. 51 (1983) 1135

[9] P. Chiappetta, J.Ph. Guillet, and J. Soffer, Nucl. Phys. B262 (1985) 187;

P. Chiappetta and J. Soffer, Phys. Rev. D31 (1985) 1019

[10] J. Babcock, E. Monsay and D. Sivers, Phys. Rev. D19 (1979) 1483

[11] European Muon Collaboration, report at Bari Conference, July, 1985

[12] J.F. Owens, Phys. Lett. 76B (1978) 85; T. Uematsu, Phys. Lett. 79B (1978) 97

[13] M.B. Einhorn, in preparation

[14] P. Chiappetta, F. Renard, J. Soffer and P. Taxil, Phys. Rev. D31 (1985) 1739; P. Chiappeta, F. Renard, J. Soffer, P. Sorba and P. Taxil, Nucl. Phys. B259 (1985) 365; B262 (1985) 495

[15] N.S. Craigie, K. Hidaka and P. Ratcliffe, Phys. Lett. 129B (1983) 310; P. Chiappetta, J. Soffer and P. Taxil, 162B (1985) 192

[16] E. Richter-Was and J. Szwed, Jagellonian Univ. preprints TPJU-7/85 (April 1985) and TPJU-9/85 (April 1985)

[17] J.C. Collins, talks given at the Supercollider physics topical conference (Eugene, Aug. 9-10, 1985); A. Mueller, talk at the recent Meeting of the Division of Particles and fields of the American Physical Society (Eugene, Aug. 12-15, 1985) 